\title{
Thromboseprophylaxe
}

\section{Erleichtertes Patienten-Management durch niedermolekulares Heparin}

- Heute werden die Patienten immer früher aus dem Krankenhaus entlassen, wie PD Dr. Martin Grünewald, Heidenheim, ausführte. Probleme, die sonst noch die Klinik gelöst hätte, verlagern sich damit auf den Hausarzt. Aber auch die Zahl der Patienten, die aus verschie-

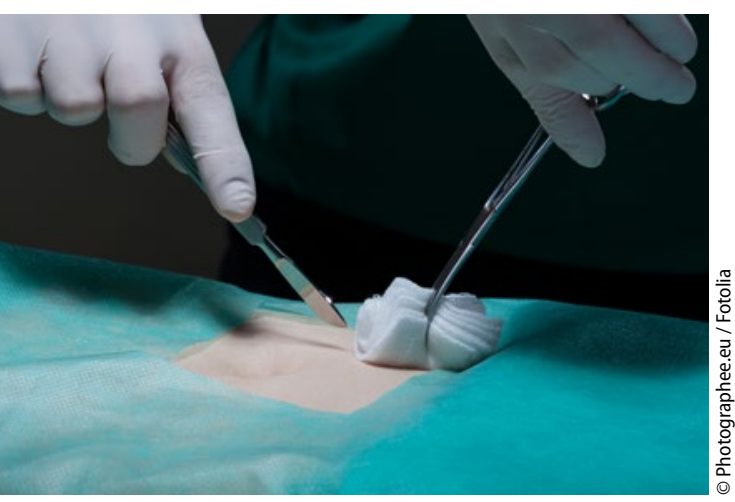

Nach Operationen ist eine wochenlange Thromboseprophylaxe notwendig. denen Gründen eine ambulante Thromboseprophylaxe benötigen, nimmt zu.

Ein hohes Thromboserisiko, das nicht an der Kliniktür endet, weisen Patienten nach orthopädischen oder abdominalchirurgischen Eingriffen auf. Nach Hüftgelenkersatz wird eine Antikoagulation über vier bis fünf Wochen, nach einem Eingriff im Bauchraum für vier Wochen empfohlen. Nach Entlassung aus nicht-operativen Abteilungen sollte eine Antikoagulation über zwei Wochen - bei individuell erhöhtem Risiko auch länger - durchgeführt werden.

Vergleichsweise einfach ist es bei der Thromboseprophylaxe onkologischer Patienten. Wegen des meist noch anhaltenden Thromboserisikos benötigen sie nach der stationären Behandlung üblicherweise eine verlängerte prophylaktische Antikoagulation, vorzugsweise mit einem niedermolekularen Heparin (NMH). Ein ambulanter Tumorpatient, dem es gut geht, braucht dagegen keine Thromboseprophylaxe, selbst dann nicht, wenn er einen Port trägt.

Wegen ihrer Indikationsbreite sind die NMH nach wie vor die Standardpräparate in der Thromboseprophylaxe und -therapie. Unter diesen schätzt Grünewald vor allem Certoparin (Mono-Embolex ${ }^{\circledR}$ ), weil es sowohl für die Prophylaxe $(1 \times$ täglich 3.000 I.E.) als auch für die Therapie $(2 \times$ täglich 8.000 I.E. $)$ eine feste Dosierung unabhängig von der Nierenfunktion und der Höhe des Thromboserisikos gibt. Die lange Halbwertszeit ermöglicht eine einmal tägliche Gabe in der Prophylaxe. Ein weiterer Vorteil ist der breite Zulassungsstatus, der auch die Thromboseprophylaxe nach akutem ischämischem Schlaganfall einschließt.

\section{Dr. Angelika Bischoff}

- Pressegespräch "Tipps vom Experten: Einfache Thromboseprophylaxe und-therapie mit Mono-Embolex ${ }^{\circledast}$ (Certoparin) in der Hausarztpraxis "; Wiesbaden, April 2016 (Veranstalter:Aspen)

\section{Schweres Asthma}

\section{Orale Steroide zurückgestuft, Biologika aufgewertet}

Orale Steroide (OCS) sind bei schwerem Asthma unverzichtbar, verursachen aber schwere Nebenwirkungen. Eine Statistik des German Asthma Net weist unter im Schnitt 50-jährigen Patienten systemische Nebenwirkungen bei $50 \%$ der Patienten aus: Hautveränderungen (27\%), Osteoporose (19\%), Diabetes (8\%) und Katarakt (6\%). Eine OCS-Dauertherapie sollte daher heute nur nach Ausschöpfung aller Optionen erfolgen, so PD Dr. Christian Geßner, Leipzig. Konkret betrifft dies Patienten, bei denen Biologika nicht indiziert sind oder Biologika das Asthma nicht kontrollieren können.

Bei akuten Exazerbationen behalten OCS ihren Platz. In schweren Fällen wird fünf Tage lang behandelt, sonst genügen kürzere Intervalle, sagte Geßner. Geßner nannte Tiotropium auf den Stufen 4 und 5, Makrolide bei neutrophilem
Asthma (allerdings würde diese dann off-label eingesetzt werden) sowie die beiden jetzt verfügbaren Biologika, die eine Differenzialtherapie ermöglichen: „Das ist im Prinzip sehr gut, wir untersuchen den Patienten genau und setzen eine spezifische Therapie ein.“

Der seit zehn Jahren bewährte IgEAntikörper Omalizumab (Xolair ${ }^{\circledR}$ ) hat seine Indikation beim allergischen Asthma mit erhöhtem IgE und sollte geeigneten Patienten nicht vorenthalten werden, so Geßner. Die Behandlung bessert das Asthma meist deutlich. Geßner zitierte mehrere Studien, bei denen $\mathrm{Pa}$ tienten unter Omalizumab neben den bekannten antiasthmatischen Wirkungen (Exazerbationsreduktion, Symptomminderung, Gewinn an Lebensqualität) ihre oralen Steroiddosen deutlich reduzieren bzw. absetzen konnten.
Auch mit dem neuen Interleukin5-Antikörper Mepolizumab (Nucala ${ }^{\circledR}$ ) können orale Steroide eingespart werden, so Geßner. Die Substanz ist indiziert zur Zusatztherapie des schweren, nicht-allergischen Asthmas, wenn erhöhte Eosinophilen-Zahlen im Blut vorliegen. Hier sollte ein Differenzialblutbild gemacht werden.

Die Frage ist: Wann soll man mit Biologika beginnen? Laut G-BA-Therapiehinweis ist Omalizumab erst nach zwei Exazerbationen einzusetzen. Dies, meinte Geßner, muss man diskutieren. Denn die neuen Leitlinien fordern, Exazerbationen und Nebenwirkungen zu vermeiden.

\section{Dr. Dirk Einecke}

- Industriesymposium „Biolocigal bei Asthma: The future is now” 57. Kongress der Deutschen Gesellschaft für Pneumologie und Beatmungsmedizin; Leipzig, März 2016 (Veranstalter: Novartis) 